\title{
Evidence for 870-GHz Phonon Emission from Superconducting Al Tunnel Diodes through Resonant Scattering by Oxygen in Silicon
}

\author{
W. Forkel, M. Welte, and W. Eisenmenger \\ Physikalisches Institut der Universität Stuttgart, Teilinstitut 1, Wiederholdstrasse 13, \\ D 7000 Stuttgart 1, Germany \\ (Received 8 May 1973)
}

\begin{abstract}
Thin-film aluminum superconducting tunnel junctions can be used to generate phonons up to $10^{12} \mathrm{~Hz}$. This is substantiated by the observation of the $29-\mathrm{cm}^{-1}$ absorption line of $\mathrm{O}^{16}$ impurities in silicon, so far only known from infrared spectra.
\end{abstract}

In earlier experiments ${ }^{1}$ it was demonstrated that superconductor tunnel diodes are devices of potential use in the investigation of phonon transport and interaction properties of materials possibly up to $10^{12} \mathrm{~Hz}$. Several experiments ${ }^{2-5}$ using tin phonon generators, however, strongly supported the assumption that most of the relaxation phonons with energies $\Omega>2 \Delta_{S n}$, the energy gap of tin (corresponding to $280 \mathrm{GHz}$ ), are reabsorbed by pair breaking, thus not leaving the generator films. Now using aluminum, a superconductor with less strong electron-phonon interaction, we have been able to observe the escape of $870-\mathrm{GHz}$ relaxation phonons, corresponding to $\Omega=24 \Delta_{\mathrm{Al}}$.

The aluminum phonon generator (bottom film, $1300 \AA$ thick; $2 \Delta_{\mathrm{Al}}=0.31 \mathrm{meV}$ at $1 \mathrm{~K}$ ) and a tin detector $\left(2 \Delta_{\mathrm{Sn}}=1.16 \mathrm{meV}\right)$ were evaporated on opposite faces of a silicon substrate (length $2.3 \mathrm{~mm}$, [111] direction) doped with $10^{18}-\mathrm{cm}^{-3} \mathrm{O}^{16}$ impurities, as roughly estimated from infrared data. ${ }^{6}$ The system Si: $\mathrm{O}^{16}$, exhibiting a far-infrared absorption line ${ }^{7}$ at $29.3 \mathrm{~cm}^{-1}(878 \mathrm{GHz})$, was used as a frequency reference, at the same time demonstrating the possibility of tunable phonon spectroscopy in the terahertz frequency range.

Because of the spectral sensitivity threshold of the tin detector which responds only to phonons of $\Omega \geqslant 2 \Delta_{\mathrm{Sn}} \simeq 8 \Delta_{\mathrm{Al}}$, we observed a steep signal increase at a generator bias voltage $V=\left(2 \Delta_{S n}+2 \Delta_{A 1}\right) /$ $e$. As we have already pointed out in a former communication, ${ }^{8}$ this confirms that the most distinct feature of the emitted relaxation phonon spectrum is a sharp high-frequency cutof $f^{2}$ at $\Omega$ $=e V-2 \Delta_{\mathrm{AI}}$, resulting from relaxation of quasiparticles injected at energies $E \leqslant e V-\Delta_{\mathrm{A} 1}$ to the gap edge $E=\Delta_{\mathrm{A} 1}$. Small spectral contributions occur, however, at higher energies resulting from processes discussed in more detail in Ref. 8 . This leads to a weak signal onset already at $\mathrm{eV}$ $\leqslant 2 \Delta_{\mathrm{Sn}}$, in agreement with the calculated phonon spectrum $N_{\text {ph }}(\Omega, V)$ which is presented in Fig. 1.

The computation of the spectrum is based on the quasiparticle decay rate according to Tewordt, ${ }^{9}$ including both relaxation and recombination with thermally excited quasiparticles, but regarding only the first decay step. Since in our experiment $e V \gg 2 \Delta_{\mathrm{A} 1}$, a useful approximation is obtained from the normal-conductor limiting case $(\Delta \rightarrow 0$, dashed lines in Fig. 1$)$.

Whereas these spectra are not monochromatic, the derivatives with respect to the generator voltage $V$ contain strong monochromatic contributions. The first derivative $d N_{\mathrm{ph}} / d V$ of the superconductor spectrum reveals a peak at $\Omega=e V-2 \Delta$, arising from the cutoff step. It was Kinder ${ }^{5}$ who first applied this fact to spectroscopy. In contrast to our experiment, Kinder's method is limited to the voltage range $2 \Delta_{\mathrm{Sn}} \leqslant e V \leqslant 4 \Delta_{\mathrm{Sn}}$ because of reabsorption in the tin generator. At $e V=24 \Delta$, as in our case with $\mathrm{Al}$ as generator, the cutoff step becomes small, but even in the normal-conductor limiting case there is still a $\delta$-function singularity in the second derivative $d^{2} N_{\mathrm{ph}} / d V^{2}$, occurring at $\Omega=e V$.

The standard lock-in technique ${ }^{10}$ is employed to obtain the first and second derivatives of the detector signal $S$ with respect to generator voltage $V$. A small, constant ac modulation is superim-

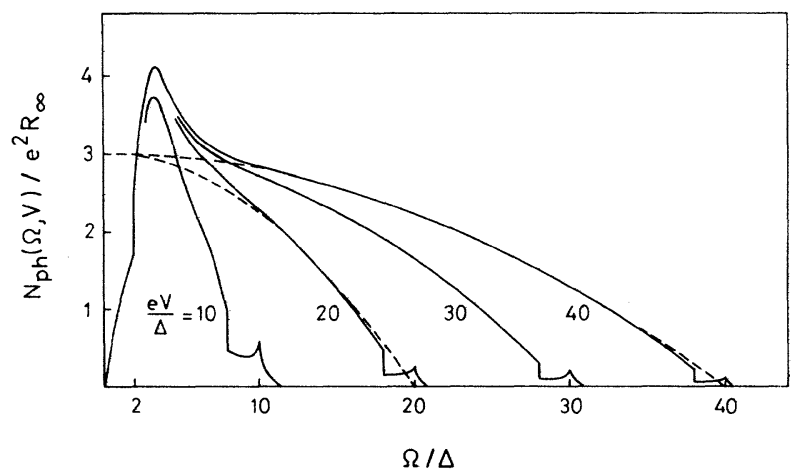

FIG. 1. Phonon emission rate in units of $e^{2} / \boldsymbol{R}_{\infty}$ for $\Delta / k T=1.6$ and for several generator voltages $V$. Dashed lines, normal-conductor limit for $T=0$. 


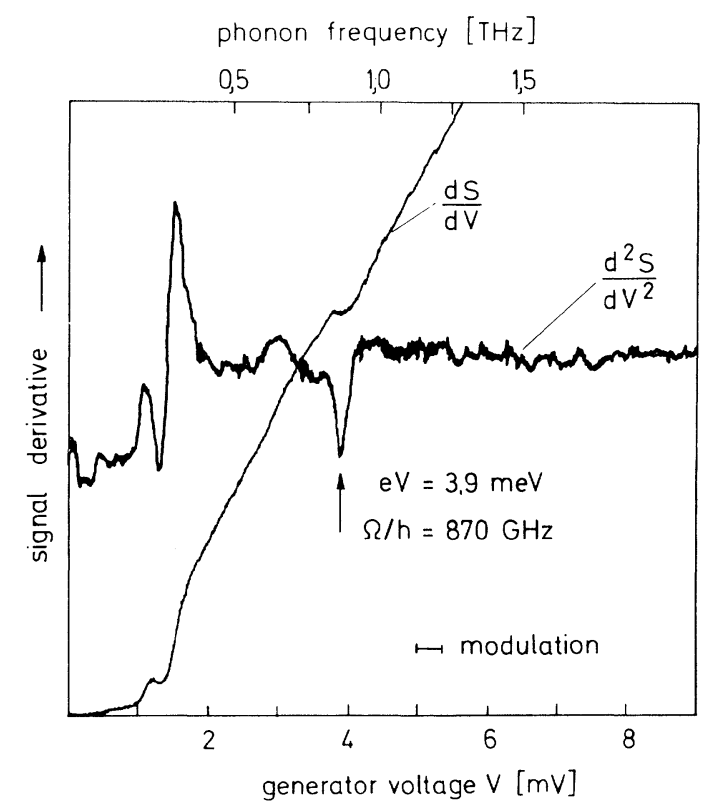

FIG. 2. First and second derivatives of detector signal versus generator voltage. Arrow indicates oxygen absorption.

posed on the dc-generator bias voltage. $d S / d V$ then is proportional to the fundamental ac component of the detector current, $d^{2} S / d V^{2}$ to the second harmonic. Experimental plots of $d S / d V$ and $d^{2} S / d V^{2}$ versus $V$ are shown in Fig. 2. Regarding first the structures below $V=2 \mathrm{mV}$ in the iirst derivative, these are readily explained as resulting from the high-frequency tail of the spectrum, in connection with the detector threshold located at $1.2 \mathrm{meV}^{8}$

The resonant structure of oxygen in silicon shows up at $3.9 \pm 0.05 \mathrm{mV}$, which, after subtracting $2 \Delta_{\mathrm{A} 1}$, corresponds to $29.0 \pm 0.4 \mathrm{~cm}^{-1}$ in accordance with the value of Hayes and Bosomworth ${ }^{7}$ within $1 \%$. This confirms that the value of the energy gap is not altered significantly by quasiparticle injection, and the cutoff step still contributes strongly to the derivative spectrum even at $V=24 \Delta_{\mathrm{A} 1}$ (the generator normal resistance was $R_{\infty}=0.6 \Omega$ ).

In our present experiment, a comparatively large modulation of generator voltage had to be used to obtain a sufficient signal amplitude, thus resulting in a spectral window of $0.3 \mathrm{meV}$. For this reason, we cannot decide whether the flat resonance structure in the first-derivative plot results from the real line shape or from finite modulation width. As a matter of principle, the resolution is only limited by the experimental tunneling density of states, which turns out to be responsible for a smearing of the cutoff step. From the generator $I-V$ characteristic, this was roughly estimated to be $10 \mu \mathrm{eV}$.

It should be worthwhile to extend the experiments up to the maximum lattice frequency of 9.5 $\mathrm{THz}$ in aluminum. No further structures or strong deviations from linearity have been observed in $d S / d V$ in the range between 1 and 4.8 $\mathrm{THz}$, where the generator films turned normal. By means of a pulse technique ${ }^{1,5}$ it should be possible to separate the LA and TA phonon modes and thus gain information on the selection rules.

We are indebted to H. J. Trumpp for suggesting the Si:O system to us and to Dr. K. Lassmann for constant interest and advice. We would also like to thank Dr. C. Irslinger (Max-Planck-Institut für Festkörperforschung, Stuttgart) for performing the infrared measurements. Miss G. Bohm showed great skill and patience in crystal preparation. The Wacker Chemitronic GmbH, Burghausen, Germany, kindly donated the silicon rods to us. Financial support from the Deutsche Forschungsgemeinschaft is gratefully acknowledged.

${ }^{1}$ W. Eisenmenger and A. H. Dayem, Phys. Rev, Lett. 18,125 (1967).

${ }^{2}$ H. Kinder, K. Lassmann, and W. Eisenmenger, Phys. Lett. 31A , 475 (1970).

${ }^{3} \mathrm{~W}$. Eisenmenger, unpublished. In experiments using a tin generator and a lead detector no signal was observed.

${ }^{4}$ R. C. Dynes, V. Narayanamurti, and M. Chin, Phys. Rev. Lett. $\underline{26}$, 181 (1971); V. Narayanamurti and R. C. Dynes, Phys. Rev. Lett. 27, 410 (1971).

${ }^{5}$ H. Kinder, Phys. Rev. Lett. 28, 1564 (1972).

${ }^{6}$ W. Kaiser, P. H. Keck, and C. F. Lange, Phys. Rev. 101,1264 (1956).

${ }^{7}$ W. Hayes and D. R. Bosomworth, Phys. Rev. Lett. $\underline{23}, 851$ (1969).

${ }^{8}$ M. Welte, K. Lassmann, and W. Eisenmenger, J. Phys. (Paris), Suppl. 10, C4-25 (1972).

${ }^{9} \mathrm{~L}$. Tewordt, Phys. Rev. 127, 371 (1962).

${ }^{10} \mathrm{D}$. E. Thomas and J. M. Rowell, Rev. Sci. Instrum. 36, 1301 (1965). 